\title{
A Personalized Radiation Exposure Management System Based on Wireless Sensor Network
}

\author{
Wonil Son and Seunghyun $\mathrm{Oh}^{*}$ \\ Department of Computer Engineering, Dongguk University Gyeongju Campus, \\ South Korea \\ jack6171@dongguk.ac.kr, shoh@dongguk.ac.kr
}

\begin{abstract}
Workers in nuclear power plants perform tasks while carrying a portable radiation instrument. The instrument sometimes works in an offline state and is used only one time, so there are difficulties in managing the amount of radiation that a worker is exposed to. Accordingly, a method of using a fixed sensor and mobile sensor is suggested to deal with radiation exposure effectively. We used a clustering-based protocol to overcome problems related to the energy of a sensor, collect radiation data around areas that workers approach to make data management easy, and check radiation levels in working areas and those of workers in real time to respond immediately to abnormal levels.
\end{abstract}

Keywords: Wireless sensor network, radiation, exposure, nuclear plant, clustering, sensor. 


\section{Introduction}

Recently, there has been an increase in interest in safety and radiation of nuclear power plants since the Fukushima Nuclear Power Plant Accident(Akashi, 2012; Rakahashi, 2014). It is difficult to effectively manage risk areas such as factories, which is highly likely to cause serious accidents. In nuclear power plants, a small mistake could result in massive damage. In Korea, there are many nuclear power plants in operation. Therefore, we are highly interested in running nuclear power plants safely and protecting the public and plant workers from radiation exposure.

Currently, some domestic nuclear plants are using a radiation monitoring system (RMS)(Park et al., 2012) and working through central processing based on wired communication. Workers in the facilities carry one-time radiation measuring devices and perform their tasks.

Workers need to respond instantly in the facility, so they should be allowed to supervise, monitor and handle accidents such as radiation leakage on site and in real time. Therefore, a system is suggested based on the QualNet simulation software (Jaikaeo and Shen, 2005) because testing in a real nuclear reactor is restricted. The system can improve the work effectiveness of workers in nuclear reactors and manage radiation leakage and individual radiation exposure. It identifies their risk factors in real time through sensor networks to respond effectively to radiation emergencies by discovering them in the beginning. The system helps construct a web server so that nuclear workers can easily check radiation levels in real time and build a database so that it becomes easier to figure out accumulated radiation exposures and levels in working areas.

In the past decades, many sensor network-based monitoring systems are suggested and shows effectiveness of the sensor networks system (Tuna et al., 2012; Aznoli and Navimipour, 2017). The system enables the development of a sensor network-based monitoring system that identifies risk factors such as abnormal radiation levels along the route of workers and manages related risks. In addition, it helps solve energy efficiency-related problems caused by a wireless sensor through the LEACH (Low-Energy Adaptive Clustering Hierarchy) protocol (Heinzelman et al. 2000). The system enables individual workers to deal with radiation exposure themselves and check radiation levels in working areas of nuclear reactors, so they can take actions against early radiation leakage immediately.

This paper is organized as follows. In Section 2, we describe previous research. In Section 3, we describe the design of the system and in section 4, we show its realization and test results. Finally, we conclude this paper in Section 5.

\section{Related Studies}

Risk area-monitoring smart robot research suggests that an embedded and wireless sensor module enables monitoring of risk areas (Koo and Baek, 2013). However, robot monitoring makes sophisticated control and taking measures instantly against 
abnormal radiation levels difficult. Our study shows that elaborate control and immediate actions are possible because human workers themselves tackle problems.

Studies of real-time radiation monitoring in nuclear reactors present a wireless sensor network (wireless sensor network) system that monitors radiation levels in real time in nuclear plants (Gomaa, 2014). There have always been studies on energy-effectiveness-related problems, but there has been no research about the energy effectiveness of a wireless sensor. Our study shows that using the LEACH protocol with high energy efficiency can lead to an increase in energy efficiency.

Research on a mobile wireless monitoring system using smartphones enabled the development of a portable radiation detector after the Fukushima accidents occurred (Ishigaki et al. 2013). However, the performance of radiation detectors is low because portability is considered more than accuracy. The previous system is dependent on the state of the network because it uses wireless networks and GPS for location information. Our suggested system can improve portability and accuracy through an arrangement of a fixed sensor and communication with a mobile sensor.

Currently, the data collected in nuclear reactors are transmitted to an emergency disaster prevention system in real time. RMS is used to transmit the data to the emergency disaster prevention system. However, RMS requires much maintenance human power because of communicating computers and peripheral equipmentrelated problems and needs periodic investment to operate computer equipment because of high prices.

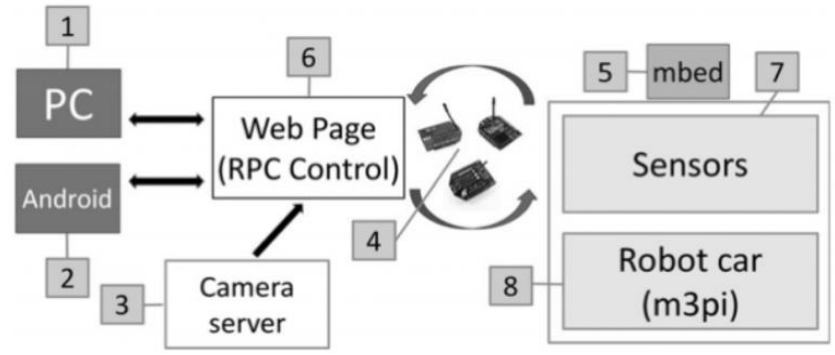

Fig. 1. Observation of Dangerous Region Using Smart Robot System

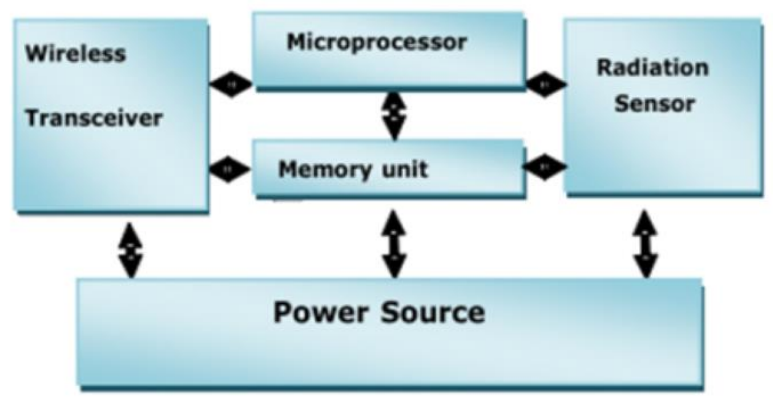

Fig. 2. Real-time Radiological Monitoring of Nuclear Facilities Using ZigBee Technology 


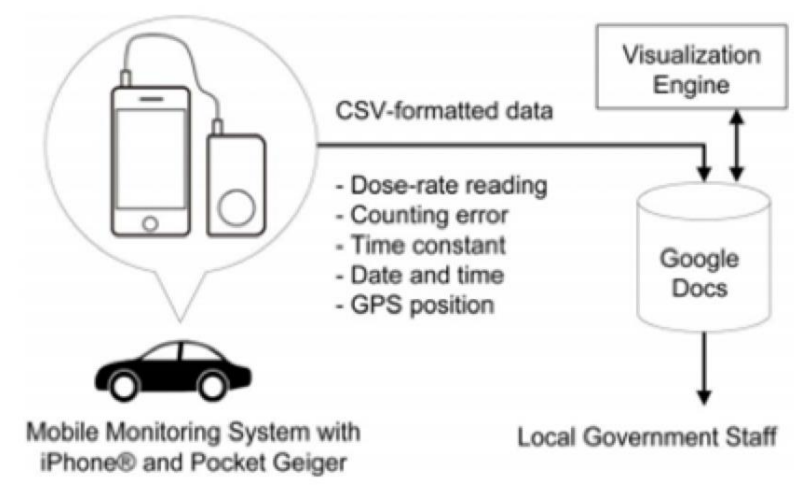

Fig. 3. Development of Mobile Radiation Monitoring System Utilizing Smartphone and its Field Test in Fukushima

In addition, nuclear workers in the facilities carry a one-time radiation measuring device and proceed with their work. Data collected within the facilities are distributed to an emergency disaster prevention system in real time. Our study shows that maintenance difficulty and high prices can be solved if the wired sensor is changed to a wireless sensor and the wireless LEACH protocol is used. Nuclear workers could easily check their radiation levels and efficiently manage their working areas.

\section{A Design of the Individual Radiation Exposure Monitoring System}

We used a simulation tool, QualNet, to conduct an experiment in this research. We set the preference of QualNet to that of a nuclear reactor and constructed an external API, DB server, and Web server. We realized a practical system by connecting an external server and the QualNet simulation tool via socket communication, transmitting an outside work order to QualNet, and storing simulation data as real data and monitoring them. This enabled us to develop a work-order management function for each worker and to manage individual radiation exposure.

Workers can monitor the amount of radiation in the plants via the Web. We set radiation levels in the simulation based on those provided in real nuclear reactors and made radiation leaks constantly. We conducted the simulation by raising the levels temporarily to check the results if there are abnormal radiation levels.

The system is largely composed of an object-based experiment model comprised of the Web and DB Server, Agent, and a simulation-based model comprised of a QualNet Socket API connecting an object-based model with a simulation-based model and the QualNet Simulator, as shown in Fig. 4. When workers try to log in, the values of $\mathrm{DB}$ and those of jsp are matched, which are connected to a log in session and enable log in. Then workers can order tasks on the Web. QualNet Socket APIWork orders are transmitted from the Web to DB, and values of work 
orders are delivered to the JAVA Agent. When there is an exchange of sensor data and status between the simulation-based experiment model's Agent and QualNet, the result values are provided to workers on the Web via the Agent.

A method of identifying data when workers follow work orders, move during work, or do not work is shown in Fig. 5. When nuclear workers use smartphones to order tasks for the movement scenario in Fig. 5 before starting to work, order data passes through DB and are transmitted to the Agent. They end up being delivered to fixed nodes arranged along the route of workers. Once all order data are delivered, workers can see the connection to QualNet on the Web. Then while working, workers can confirm the data of nodes arranged along the route going from QualNet to the Agent on the Web, and the data transmitted to the Agent can be stored in DB.

When workers finish their tasks and do not work, if they search for a certain date on the Web, they can find out their radiation exposure on that date. If they ask for a certain sensor in working areas, they can check radiation levels in that sensor. The node arrangement in a simulator is referred to a real plants' arrangement, and nodes are arranged within the scope of communication of fixed nodes after the size and width of real nuclear plants are considered. The size of a real nuclear plant is approximately $300 \mathrm{M} \times 300 \mathrm{M}$ and considering that $95 \mathrm{M}$ is the average available communication range of the wireless sensor node, ZigBee [9], nodes are placed and applied in the simulator.

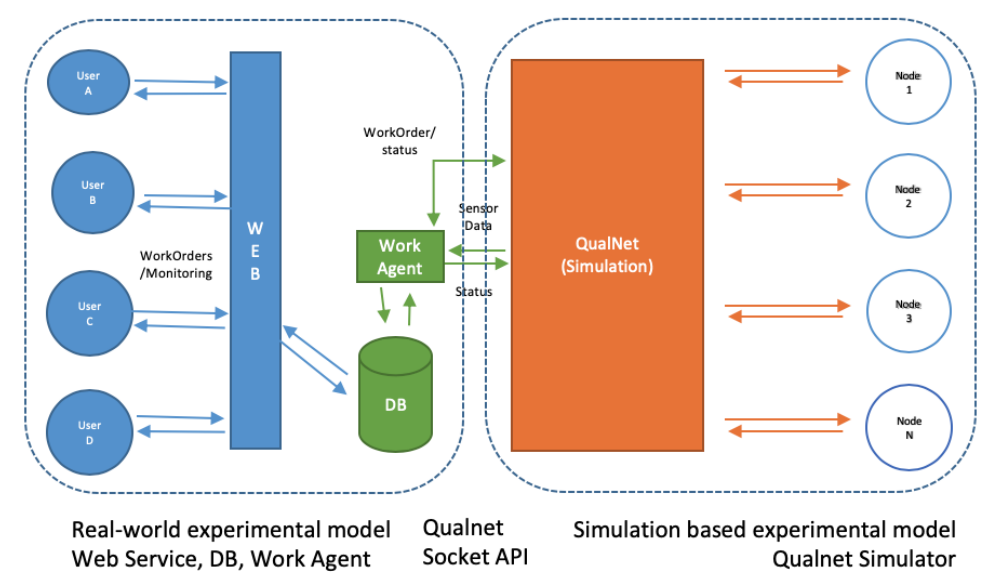

Fig. 4. System Configuration for Real-time Individual Management 


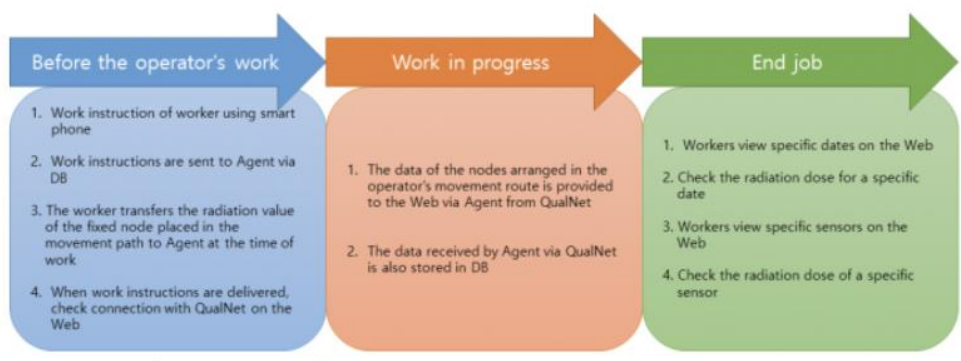

Fig. 5. Scenario for System Operation
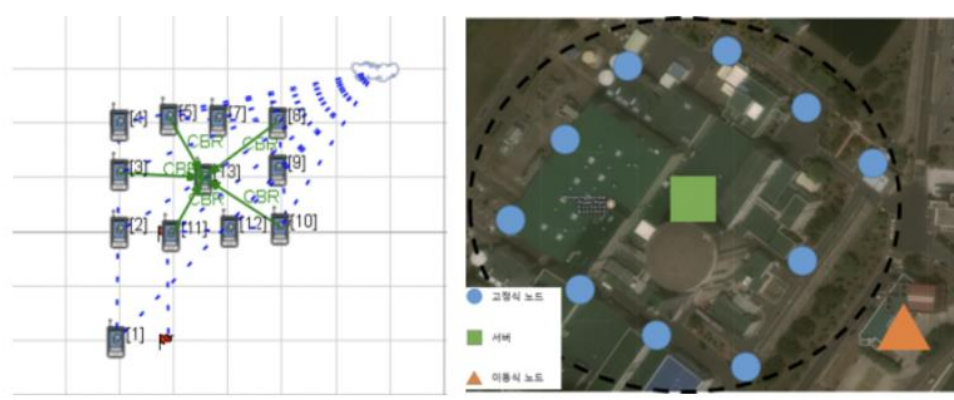

Fig. 6. Node Arrangements Based on Real Nuclear Plants

We placed fixed nodes (No. 2-13) and a mobile node (No. 1) in a simulator. We set the mobility in a mobile node after considering a worker's working speed and walking speed. In terms of mobility, we make node No. 1 move consecutively along the dotted line in Fig. 6 and the other nodes are set the wireless preference marked as a circle in the Fig. 6 because all are comprised of a wireless sensor node. Thus, we set the simulation environment and configured a work-order-based system, which enables workers to check node data and to search data such as a certain date on the Web, as shown in Fig. 7. 


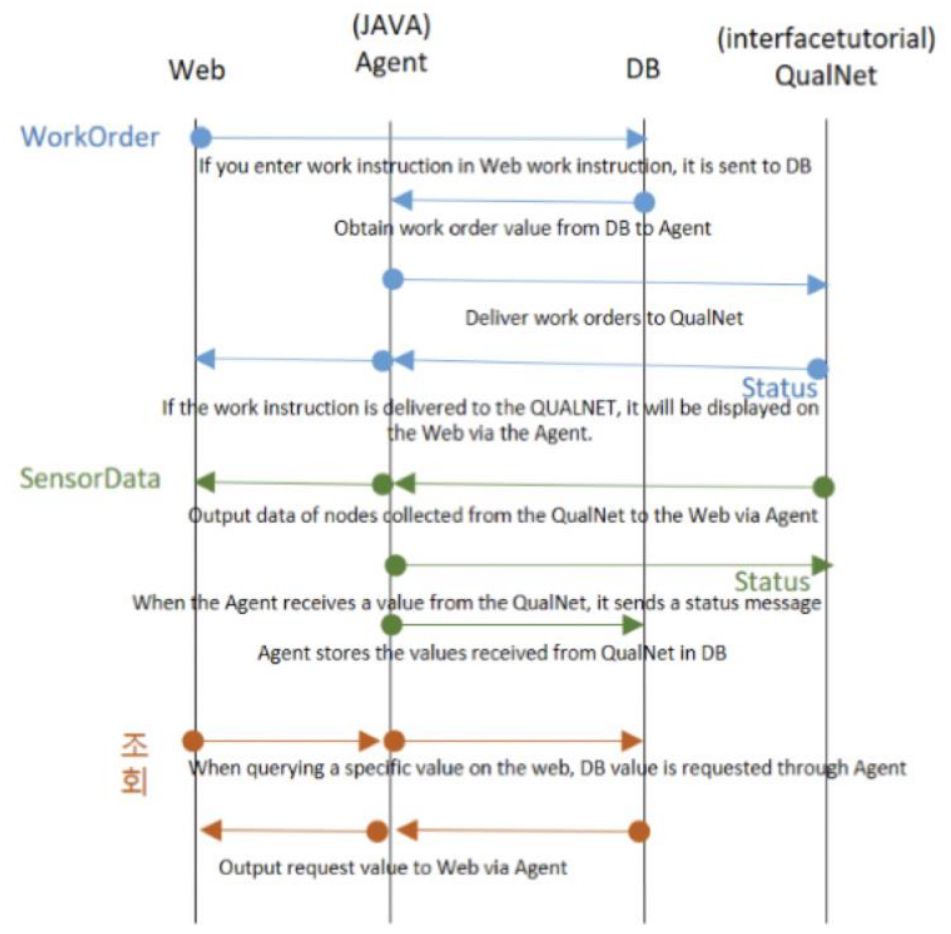

Fig. 7. Scenario Diagram for Individual Radiation exposure Management Based on Work Orders

Our system is largely divided into three structures: Work Order, Sensor Data, and Web monitoring. A work order is a necessary process for efficiently managing individual radiation amounts in working areas and individual radiation exposure by making workers register the work date, area, and other information before they begin to work. All work-order-related data is stored in DB, so workers can always check a certain date and areas. Sensor data are substantially including a place where data of sensor collected, time information, and sensor value. Web monitoring is a service for efficiently managing the radiation exposure of workers and radiation levels in working areas. When workers carry their smartphones and network communication is connected, they can check their radiation exposure on a certain date and in particular areas anytime and anywhere

\section{Experiments and Discussion}

We realized the radiation measurement system based on wireless sensor networks in a simulation. We changed data values of sensors into radiation levels in the system. We also made a database that manages and stores values and a Web system that workers can monitor. We connected the system and simulator using a method of interface connection outside the simulator QualNet for our experiment. For smooth 
communication between the database, Web, and simulators, we introduce the Agent.

We confirmed the size of nuclear reactors and work speed of workers and set the simulation based on a real working environment to configure the same environment as a real nuclear plant. We placed nodes in a simulator for smooth communication between nodes by applying the size of real nuclear reactors and the communicable distance of real nodes. We applied total work hours to the simulator by considering the moving speed of workers and expected work hours. Finally, we made a user interface for monitoring so that workers can manage data as conveniently as possible. We made workers carry mobile sensors given that they move in working areas of nuclear plants and installed fixed sensors within the areas of the plants to measure radiation levels.

Installable and portable sensors comprise a wireless sensor network. Therefore, we can fix high-cost maintenance problems by changing from wired to wireless communication in nuclear plants. We solved connection and energy-related problems of wireless sensor networks by using a clustering protocol, LEACH. The LEACH protocol is suitable for energy management of sensors because the protocol is developed to improve the energy efficiency of sensors.

We managed the radiation levels for each of the locations by installing fixed sensors in a building or particular location within nuclear plants. The smartphones of workers have a combination of a mobile radiation sensor, warning registration, and alarm application. Their smartphones are able to collect and monitor radiation vales of mobile sensors measured along the movement path of workers and radiation levels measured by fixed sensors. When workers move, the smartphones are asked to give warnings and emergency alarms. We added the conception of a sink node to a fixed node, integrated data values of fixed sensors, and made the fixed nodes send the data to the Agent.

The Agent plays a role in delivering data from mobile sensors and fixed sensors. Mobile sensors integrate, manage, and monitor the measured radiation levels and the values measured by fixed sensors located along the movement path. According to the results of work orders, mobile sensors are required to find data on radiation levels from mobile sensors located along the path of mobile sensors. Sensors and smartphones are interactive to measure values of sensors, which should be open to users and tell them the radiation levels of nuclear reactors and radiation exposures of workers during work.

We created a Web environment to enable workers to see their radiation exposures and radiation levels in working areas easily. When there are work orders on the Web, the work-order-related information is delivered from the Agent to QualNet. Based on the delivered work order information, a simulation was conducted on QualNet, and the generated data are transmitted through the Agent to the Web to enable workers to see information from sensors and radiation exposures between the tasks of workers. During work, workers and users can figure out the time, values, 
and location data of sensors on the Web. Red means abnormal, and blue is normal. In addition, when workers are not present, if they want to check data values of sensors for a particular year, month, and day, they can find them as shown in Fig. 8.

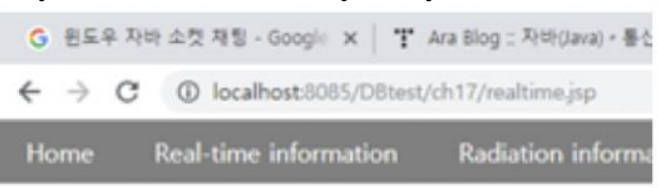

\begin{tabular}{|l|l|l|l|l|}
\hline & SENSOR1 & SENSOR2 & SENSOR3 & SENSOR4 \\
\hline TIME & & & & \\
\hline VALUE & 105 & 52 & & \\
\hline LOCATION & & & & \\
\hline
\end{tabular}

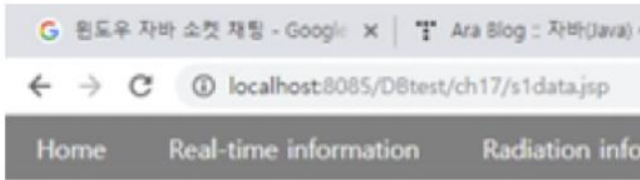

HOME

- vear value omonth value oday value

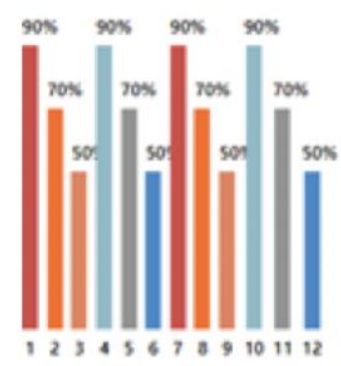

Fig. 8. Configuration of Real-time Sensor Information

Workers can check the cumulative data of sensors and real-time data during the total period of their working hours, as shown in Fig. 9. The radiation exposure of a worker can be searched by month or a particular day, and radiation levels can be found in the same way. 


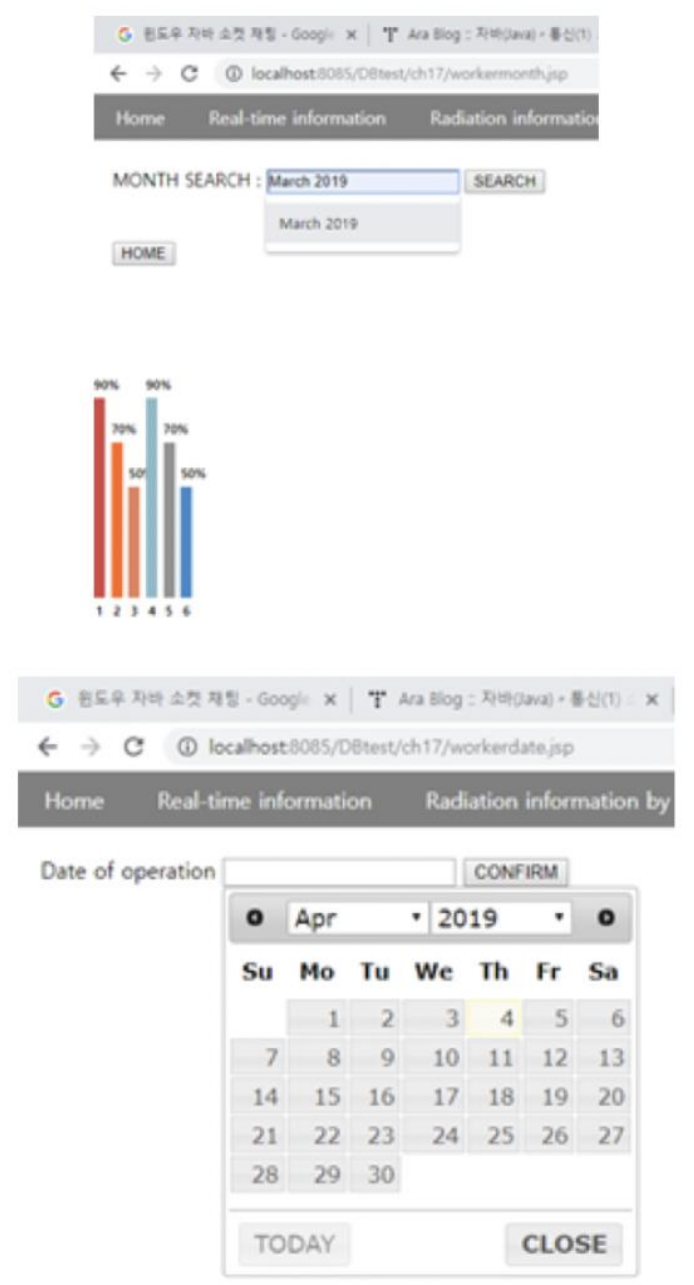

Fig. 9. Configuration of Radiation Information

We made a simulation for the time and working environment with the same configuration as real nuclear plants, as shown in Fig. 11. Nodes were placed within the range of communication between nodes and nuclear reactors, and working time was adjusted by considering the size of nuclear plants and the average moving speed of workers. Energy consumption values were compared with those of the AODV protocol, which is mostly used in existing wireless sensor networks. When LEACH is used, less energy is consumed than when the AODV protocol is used in transmission and receiving modes. The energy consumed by the LEACH protocol is $30-40 \%$ less than that of the AODV protocol in a transmission mode, and there are also nodes that are never participated as shown in Fig. 10. The energy consumption of the LEACH protocol is also 30-40\% less than that of the AODV protocol in a receiving mode as shown in Fig. 11. 


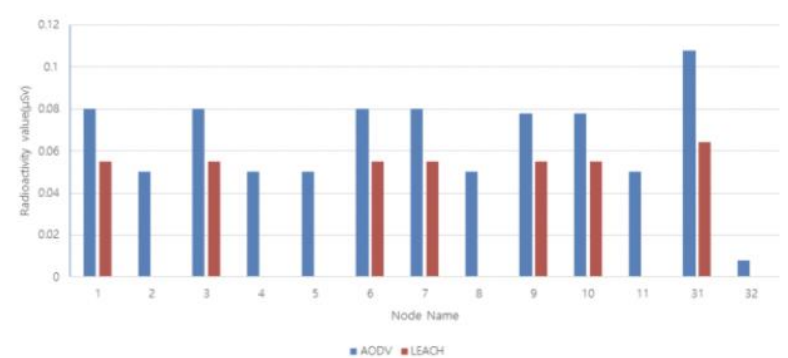

Fig. 10. Consumption Energy Comparison in Transmission Mode per Routing Protocol

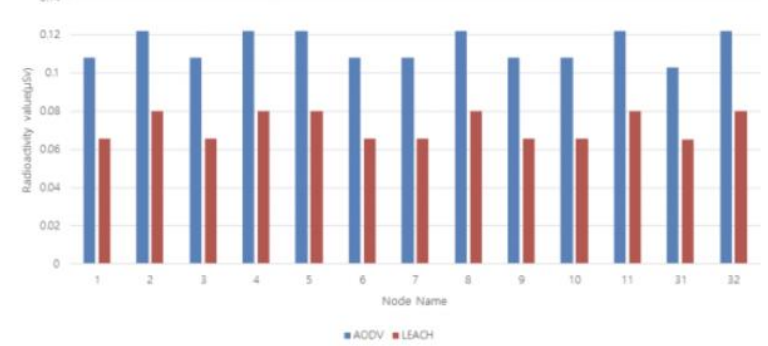

Fig. 11. Consumption Energy Comparison in Receiving Mode per Routing Protocol

Workers' tasks were also performed in a simulated environment. Based on radiation levels of real nuclear reactors, workers select working areas in the simulation and individually set the working date, area, data, and route of a working area. The system configuration for working is shown in Fig. 12.

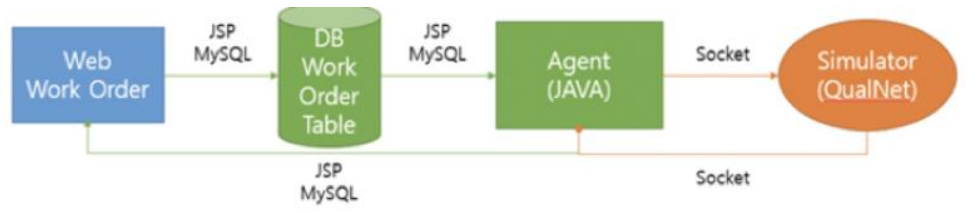

Fig. 12. Data Flow of WorkOrder Processing

When a work order is applied on the Web, JSP and MySql are used to transmit radiation levels to the work order Table of DB and send it to the Agent. Then the Agent sends the values to the simulator through socket communication, and vice versa. Finally, the Agent shows radiation levels to workers on the Web. Workers register working information between working orders, which are nodes located on the route of working areas and simulate the registered nodes to see the radiation exposures of nodes 1, 3, 6,7, 9, and 10, as shown in Fig. 13. Node 32 in the front is a mobile node that workers carry, and radiation exposures when workers are present in working areas can be seen in Fig. 14. The graphs show the radiation exposure of a mobile sensor (node 32) of one worker during work. If other workers perform their tasks in the same area, their radiation exposure levels are added to the graph. 


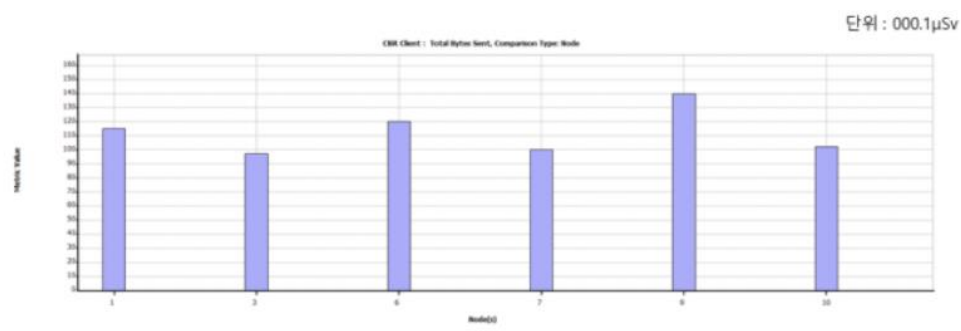

Fig. 13. Radiation Exposures on Fixed Nodes on Tasks by Workers

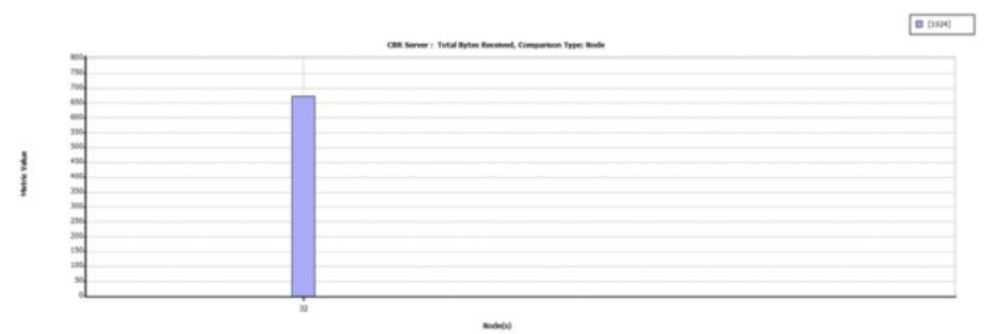

Fig. 14. Radiation Exposure on Mobile Sensors on Tasks by Workers

Likewise, values measured at fixed nodes and mobile nodes are transmitted through the Agent to $\mathrm{DB}$, and the transmitted data are sent to the Web so that workers can conveniently see values such as sensor values and radiation exposure, as shown in Fig. 15. Additionally, if they input a certain date to find the radiation exposures of workers, they can see radiation levels emitted from nodes that were registered on that day.

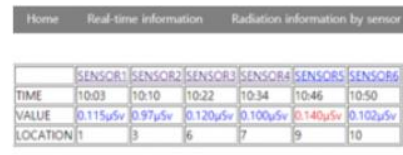

Fig. 15. Radiation Exposures of Sensors on the Web

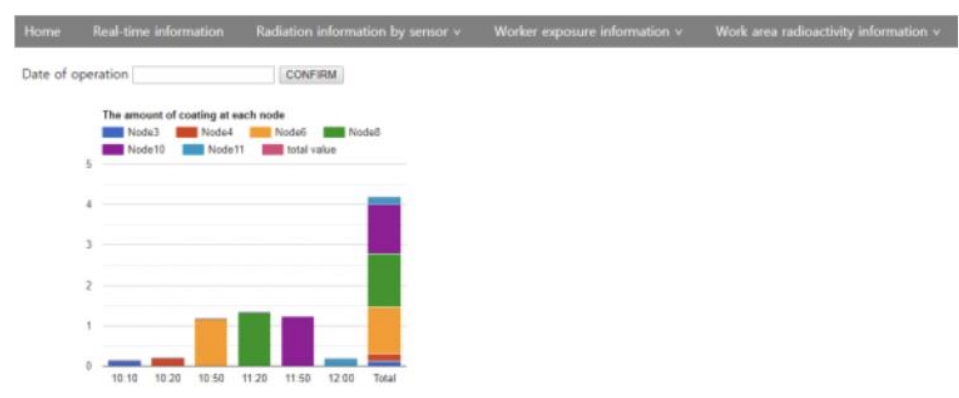

Fig. 16. Accumulated Radiation Exposures of Workers on the Web 


\section{Conclusion}

This study suggested how to effectively manage nuclear plant workers' radiation data via wireless sensor networks. Sensors were located at fixed nodes in working areas of nuclear plants and on mobile nodes that workers carry. The LEACH protocol was used so that fixed sensors and mobile sensors are clustered when workers are present in the plants, resulting in overcoming the energy problems of sensors efficiently. Personal data are added in current sensor networks so that radiation levels in working areas and workers' radiation exposures can be easily searched on the Web, and abnormal radiation levels can be immediately responded to in the areas.

\section{Acknowledgments}

These should be brief and placed at the end of the text before the references.

\section{References}

Akashi M. (2012). Fukushima Daiichi Nuclear Accident and Radiation Exposure. JMA Symposium on Health Policy "Disaster Medicine and Medical Associations" JUMAJ, 55(5), 393-399.

Rakahashi S. (2014). Radiation monitoring and dose estimation of the Fukushima Nuclear Accident. Tokyo.

Park, W. M, Lee, D. Y., Park, J. C., Hwang, I. K., Kim, J. Y., Choi, J. G. and Lee, G. Y.,(2012). Maintenance of Radiation Monitoring System (RMS) in the nuclear facility (KAERI/RR--3363/2010). Korea, Republic of.

Jaikaeo, C. and Shen, C. C. (2005). Qualnet tutorial.

Heinzelman, W., Chandrakasan, A and Balakrishnan, H. (2000). Energy-Efficient Communication Protocols for Wireless Microsensor Networks. IEEE Proceedings of the Hawaii International Conference on Systems Science, 1-10.

Koo K.-W. and Baek D.-H. (2013). A Study of Smart Robot Architecture and Movement for Observation of Dangerous Region. Journal of Korean Institute of Fire Science and Engineering, 27(6), 83-88.

Gomaa R.I., Shohdy I.A., Sharshar K.A., Al-Kabbani A.S. and Ragai H.F. (2014). Real-Time radiological monitoring of nuclear facilities using zigbee technology. IEEE Sensors Journal, 14(11), 4007-4013. 
Ishigaki Y., Matsumoto Y., Ichimiya R. and Tanaka K. (2013). Development of Mobile Radiation Monitoring System Utilizing Smartphone and Its Field Tests in Fukushima. IEEE Sensors Journal, 13(10), 3520-3526.

ZigBee Alliance. https://www.zigbee.org/

Tuna, G., Gulez, K., Mumcu, T.V. and Gungor, V.C.(2012). Mobile robot aided self-deploying wireless sensor networks for radiation leak detection. Proceedings of the 5th International Conference on New Technologies, Mobility and Security (NTMS) IEEE, 1-5.

Aznoli F. and Navimipour N.J. (2017). Deployment Strategies in the Wireless Sensor Networks: Systematic Literature Review Classification and Current Trends. Wireless Personal Communications, 95, 819. 